\title{
BUILT-UP AREA DETECTION BASED ON SUBSPACE PROJECTIONS USING POLARIMETRIC SAR DATA
}

\author{
R. Bordbari ${ }^{\text {a*}, ~ Y . ~ M a g h s o u d i ~}{ }^{\text {a }}$, M. Salehi ${ }^{\text {a }}$ \\ ${ }^{a}$ Department of Remote Sensing, Faculty of Geomatics Engineering, K.N. Toosi University of Technology, Iran - \\ rbordbari@mail.kntu.ac.ir, ymaghsoudi@kntu.ac.ir, msalehi@mail.kntu.ac.ir.
}

KEY WORDS: Built-up, Detection, Polarimetric SAR, Helix, Scattering Mechanisms

\begin{abstract}
:
The task of detecting and identifying objects remotely has long been an area of intense interest and active research. Active sensing of objects with radio waves is a whole new domain of target detection which is made available by radar remote sensors. Land cover/use information extraction is one of the most important applications of radar remote sensing, especially in urban areas. In this paper, we take a new look at the built-up area extraction problem in polarimetric SAR (PolSAR) data and assume canonical scattering mechanisms as our signal sources which combination of them with appropriate weight fractions formed a scattering vector of each pixel. The set of the scattering mechanisms is divided into two groups: the scattering mechanism of built-up area, and non-objected scattering mechanisms. Then, we describe a technique which simultaneously annihilates the effect of non-objected scattering mechanisms, and detects the presence of a scattering mechanism of interest. The experimental results on several quad-polarimetric datasets show the significant agreement with expected results, while saving computational complexity.
\end{abstract}

\section{INTRODUCTION}

The use of radar remote sensing to explore urban environments and retrieve spatial, temporal, and environmental characteristics of urbanized areas is becoming an important research line. Radar remote sensors, which can work day and night and are nearly unaffected by weather and atmospheric conditions, play an increasingly important role for Earth and other planet monitoring in both global and regional scales. The improved performance of new radar sensors with finer resolutions and additional channels (PolSAR) increases the sensitivity to more complex structures and gives more physical sense to urban scattering analysis. Fully polarimetric information is sensitive to the scattering mechanisms related to target responses during a backscattering procedure. Full polarization acquisition can enhance the radar capability in scattering mechanism understanding and target parameters retrieval such as material, shape, and orientation. PolSAR systems can acquire both the geometrical and the physical information of a scatterer, and store them into the scattering matrix (for a single scatterer) or coherency matrix (for a mixed scatterer) (S.-W. Chen, et al. 2014). An understanding of the scatterer is then obtained by processing these two matrices.

Scattering mechanism understanding is a bridge between the collected data and the real applications. Generally, scattering mechanisms are determined by a set of factors such as radar frequency, radar bandwidth, illumination direction, and target dielectric and geometric properties (J.-S. Lee and E. Pottier 2009). Although the basic theory of radar polarimetry has been well established (J.-S. Lee and E. Pottier 2009), scattering mechanism modeling and interpretation are still ongoing and how to effectively extract robust and useful information from collected data is still challenging. In the field of PolSAR data utilization, man-made target detection and built-up area extraction in urban areas is one of the most important topics. Most of built-up areas are composed of many strong scattering centers with different orientations, which make the whole structure of the man-made targets not to be reflection symmetric (S. Cloude 2010). There is a theoretical scattering mechanism, namely helix, that fits the reflection asymmetry condition and valid in heterogeneous areas, such as complicated shapes targets and built-up areas (S.-W. Chen et al. 2014). Helix scattering is a general scattering mechanism which appears in an urban area whereas disappears for almost all natural distributed scattering. It can distinguish man-made target from natural targets well but cannot divide one type of man-made target from another kind.

The aim of this paper is built-up area detection exploiting a novel feature vector formalism generated from the coherency matrix. The feature vectors of canonical scattering mechanisms are defined firstly. Then, the feature vectors of the canonical scatterers constitute columns of the signal matrix. This matrix is, then, divided into two groups: one for the helix scattering mechanism and another for the non-objected scattering mechanisms. In this study, the detection technique is based on subspace projection which can be implemented in two stages in cascade: reduction of speckle effect and annihilation of nonobjected scattering mechanisms in the first stage, followed by detecting helix scattering mechanism in the second stage. In this case, as it will be shown, with the elimination of the nonobjected mechanisms, the detectability of the desired mechanism can be therefore enhanced.

\footnotetext{
* Corresponding author
} 


\section{PROBLEM FORMULATION}

When a scatterer is illuminated by an electromagnetic wave, the description of the polarization state of the backscattered wave can be represented by a $2 \times 2$ Sinclair scattering matrix or, equivalently, by a scattering vector. Due to the ease of interpreting the physical scattering mechanism, the Pauli basis set is largely exploited to convert scattering matrix into scattering vector. Subject to reciprocity condition, the offdiagonal terms of the scattering matrix are equal, and the scattering vector in the Pauli basis is defined as $k=1 / \sqrt{2}\left[\begin{array}{lll}S_{H H}+S_{V V} & S_{H H}-S_{V V} & 2 S_{H V}\end{array}\right]^{T}$. The measured scattering is the coherent superposition of the individual scattering matrices within the resolution cell. Therefore, the effects connected to distributed scatterers cannot be analyzed by the measured scattering matrix. These scatterers can only be characterized statistically by the second-order polarimetric representations. In this context, a second-order descriptor can be estimated as $[T]=\left\langle k k^{\dagger}\right\rangle$, where the superscript $\dagger$ stands for complex conjugation and transposition, and $\langle$.$\rangle is used here as$ the finite averaging operator for speckle reduction.

The spatial coverage of each pixel in PolSAR image may encompass several different scatterers, each with a unique scattering mechanism. In this case, the scattering matrix is affected by the individual scattering mechanisms of each scatterer, and the pixel can be termed mixed. In our proposed algorithm, we have a mixed pixel represented as a linear combination of $m$ canonical scattering mechanisms with appropriate weight fractions. The weight of each mechanism is proportional to the fraction of the pixel's polarimetric scattering information belongs to that canonical scatterer. The $l$ dimensional feature vector of observed pixel $r(x, y)$, can be described by

$$
r(x, y)=\omega_{1} s_{1}+\omega_{2} s_{2}+\cdots+\omega_{m} s_{m}
$$

where $l$ is the dimension of feature space, $(x, y)$ is the spatial position of the observed pixel, $s_{i}$ is the polarimetric backscatter of $i$ th scatterer, and $\omega_{i}$ represents the fraction of $i$ th scatterer's polarimetric backscatter present in the pixel. However, due to the coherent processing of the scattered signals in PolSAR images, they are highly susceptible to speckle effects (S.-W. Chen, et al. 2014). The presence of speckle not only reduces the ability of a human observer to resolve fine detail, but also complicates the image analysis. From the image processing point of view, speckle can be characterized statistically by a multiplicative noise model, in the sense that the speckle noise level is proportional to the scene signal level. The multiplicative noise model has been derived and verified for 1-look or multilook processed PolSAR images (J.-S. Lee and E. Pottier 2009).

Let $r_{i}$ denote the intensity level of an arbitrary pixel in the image, $z_{i}$ and $v_{i}$ denote the signal and the multiplicative noise, respectively. The pixel level can be written as $r_{i}=z_{i} v_{i}$, where $v_{i}$ is a measure of speckle strength and has unity mean and standard deviation $s_{i}$. It is assumed that $v_{i}$ is statistically independent from $z_{i}$ and $v_{j}(i \neq j)$. Lee et al. in (J.-S. Lee and K. Hoppel 1992) showed that this multiplicative noise model is equivalent to a signal-dependent additive noise model. Since $v_{i}$ has unit mean, (1) can be written as $r_{i}=z_{i}+n_{i}$, where $n_{i}=z_{i}\left(v_{i}-1\right)$ is zero-mean signal-dependent additive noise, and its variance is (J. Gu, et al. 2004)

$$
\sigma_{n_{i}}^{2}=E\left\{\left|r_{i}\right|^{2}\right\} \frac{\sigma_{i}^{2}}{\sigma_{i}^{2}+1}
$$

Hence, the variance of the additive noise $n_{i}$ is approximately proportional to the average square intensity of the pixel, and this signal-dependent noise of a given pixel is independent from that of any other pixel. The speckle noise is uncorrelated with polarimetric SAR channels and by extracting the dominant information, the speckle effect may be reduced (J. Gu, et al. 2004). The equation (1) can be rewritten as

$$
r(x, y)=S \omega(x, y)+n(x, y)
$$

where the matrix $S=\left(s_{1}, \ldots, s_{m-1}, s_{m}\right)$ is a $l \times m$ signal matrix, $\omega$ is a $m \times 1$ vector where the $i$ th element represents the fraction of the $i$ th canonical scattering mechanism present in the pixel, and $n$ is an $l \times 1$ error vector accounting for signal-dependent additive noise. If the medium is reciprocal, the polarimetric backscatter of a mixed pixel can be described by means of independent elements of its coherency matrix and one can construct a feature vector

$$
\begin{aligned}
r= & {\left[r_{1}, r_{2}, r_{3}, r_{4}, r_{5}, r_{6}, r_{7}, r_{8}, r_{9}\right]^{T} } \\
= & {\left[\left\langle\left|k_{1}\right|^{2}\right\rangle,\left\langle\left|k_{2}\right|^{2}\right\rangle,\left\langle\left|k_{3}\right|^{2}\right\rangle, r e\left\langle k_{1}^{*} k_{2}\right\rangle, \operatorname{im}\left\langle k_{1}^{*} k_{2}\right\rangle,\right.} \\
& \left.\quad \operatorname{re}\left\langle k_{1}^{*} k_{3}\right\rangle, \operatorname{im}\left\langle k_{1}^{*} k_{3}\right\rangle, \operatorname{re}\left\langle k_{2}^{*} k_{3}\right\rangle, \operatorname{im}\left\langle k_{2}^{*} k_{3}\right\rangle\right]^{T} .
\end{aligned}
$$

This feature vector can be used for representing completely and uniquely a partial target. This mathematical formulation is general and can be applied to any kind of scattering mechanisms; however, in this paper, the detection is optimized for detecting a theoretical scattering mechanism, namely, helix. Without loss of generality, assume that the last columns of $S$ is the helix scattering feature vector denoted by $d$ and the remaining columns are the non-objected scattering mechanisms denoted by $V_{I}=\left(s_{1}, s_{2}, \ldots, s_{m-1}\right)$. An equivalent statement of (3), which separates the desired and non-objected scattering mechanisms, is given by

$$
r=d \omega_{d}+V_{I} \omega_{I}+n
$$

For clarity, subscripts are omitted and polarimetric backscatter of a mixed pixel is written as equation (3), where $\omega_{d}$ and $\omega_{I}$ are the weights of the desired and the unwanted scattering mechanisms, respectively.

There are several scattering mechanisms in urbanized areas, for instance, even-bounce, dipole, helix, volume (dihedrals oriented at $45^{\circ}$ with respect to radar line of sight), etc. However, due to the orbital and side-looking arrangement of the radar platforms, a trihedral-like scattering mechanism appears in built-up areas. This scattering mechanism can be interpreted as layover effect which is an inherent phenomenon in urban areas. To cope with this issue, the odd-bounce scattering mechanism is only assumed as non-objected scattering mechanism.

The aim of our formulation scenario is to fit a combination of several scattering mechanisms to PolSAR observations. However, depending on the spatial resolution of the PolSAR images it is possible to present lots of scatterers in the spatial coverage of each pixel. In order to address this problem, the 
error vector $\delta$ is defined as lack-of-fit effect and interpreted as a $9 \times 1$ column vector. This model error is assumed to be an independent identically distributed (i.i.d.) Gaussian process with zero mean and covariance matrix of $\sigma^{2} I$, and the equation (5) is rewritten as

$$
r=d \omega_{d}+V_{I} \omega_{I}+n+\delta
$$

The idea is to find an optimal weight value for the objected scattering mechanism (target) and eliminate the effects of the noises, errors and non-objected canonical scatterers.

\section{BUILT-UP AREA DETECTION}

\subsection{The Speckle Effect Annihilation}

In our formulation scenario, it was assumed that there are five different canonical scatterers (standard and $45^{\circ}$ dihedrals, helix, dipole, trihedral) in the pixel area. Also the elements of feature vector of the observed pixel in (6) were assumed as being corrupted by signal-dependent additive noises, the variance of which is proportional to the square of average intensity of the pixel. Therefore, there are five linear independent directions for the feature vectors that span a 5-dimensional linear subspace. The 5-dimensional subspace can be called the "signal subspace", and the other 4-dimensional subspace is called the "noise subspace" [5].

Let $\mu_{r}$ the mean vector of the feature vector $r$. The covariance matrix of the feature vector $r$ is $C_{r}=E\left\{\left(r-\mu_{r}\right)\left(r-\mu_{r}\right)^{T}\right\}$. Since $C_{r}$ is conjugated symmetric matrix, we can apply eigenvalue decomposition to $C_{r}$, which may be expressed as $C_{r}=Q \Lambda Q^{T}$, where $\Lambda=\operatorname{diag}\left(\lambda_{1}, \lambda_{2}, \ldots, \lambda_{9}\right)$ and $\lambda_{i}$ is the $i$ th eigenvalue of $C_{r}$. Because $C_{r}$ is semi-positive definite, the nonnegative eigenvalues $\lambda_{i}$ can be arranged in decreasing order. The columns of the $9 \times 9$ matrix $Q$ consist of 9 orthogonal eigenvectors of $C_{r}$, and these eigenvectors define the basis of the 9-dimensional linear space. There are five targets in the spatial coverage of each pixel, and noise is present, thus we would have five larger eigenvalues, and four smaller ones. Thus, the matrix $Q$ can be rewritten as $Q=\left(Q_{S} \mid Q_{N}\right)$, where the $9 \times 5$ matrix $Q_{S}$ is composed of the eigenvectors corresponding to the five larger eigenvalues, and the $9 \times 5$ matrix $Q_{N}$ is composed of remaining four eigenvectors corresponding to the four smaller eigenvalues.

It can be shown that the 5-dimensional linear subspace based on the columns of $Q_{S}$ is coincident with the "signal subspace," while the subspace based on the columns of $Q_{N}$ is the "noise subspace" (J. Gu, et al. 2004). Therefore, our feature vector $r=\left(r_{1}, r_{2}, \ldots, r_{9}\right)$ can be expanded as a linear combination of the eigenvectors $q_{1}, q_{2}, \ldots, q_{9}$ and the mean vector $r=\sum_{i=1}^{9} c_{i} q_{i}+\mu_{r}$, where $\sum c_{i} q_{i}$ is the variant part of the equation and the coefficients $c_{i}$ are $c_{i}=q_{i}^{T}\left(r-\mu_{r}\right)$. In order to eliminate the "noise subspace," we make use of a projection matrix $P_{Q_{s}}$ to orthogonally project the variant part of $r$ into the "signal subspace" as

$$
\begin{aligned}
\hat{r} & =P_{s}\left(\sum_{i=1}^{9} c_{i} q_{i}\right)+\mu_{r} \\
& =\sum_{i=1}^{5} c_{i} q_{i}+\mu_{r},
\end{aligned}
$$

where $P_{S}=\left(Q_{S}\left(Q_{S}^{T} Q_{S}\right)^{-1} Q_{S}^{T}\right)$ is the orthogonal projection operator which reduces the contribution of the "noise subspace" to zero.

\subsection{The Non-objected Mechanisms Annihilation and the Objected Scattering Mechanism Detection}

In order to effectively extract the scattering mechanism $d$, we need to annihilate the unwanted effects of the non-objected scattering mechanisms which are presented by the columns of $V_{I}$. To achieve this goal, an operator should be formed that projects $\hat{r}$ onto a subspace that is orthogonal to the columns of $V_{I}$. In order to do so, we make use of a projection matrix which is defined by $P_{V}^{\perp}=I-V_{I} V_{I}^{\#}$, where $V^{\#}=\left(V_{I}^{T} V_{I}\right)^{-1} V_{I}^{T}$ is the pseudo-inverse of $V_{I}$. The vector resulting from multiplication $P_{V}^{\perp}$ and $\hat{r}$ will only contain energy associated with the objected scattering mechanism and lack-of-fit error. Operating on (6), we have

$$
P_{V}^{\perp} \hat{r}=P_{V}^{\perp} d \omega_{d}+P_{V}^{\perp} \delta
$$

In this approach, orthocomplement of $V_{I}$ reduces the contribution of $V_{I}$ to zero. In order to estimate the weight of the objected scattering mechanism, we take advantage of the simultaneous diagonalization (SD) transform (J. W. V. Miller, et al. 1992). This can be applied to the filtering of PolSAR image to generate one new image in which the objected signal is enhanced while the unwanted effects of the errors are suppressed. The aim is to find the $1 \times 9$ operator $h^{T}$ based on maximizing the ratio of the energy of the objected scattering mechanism to the error energy after filtering. Operating on (8) we have

$$
h^{T} P_{V}^{\perp} \hat{r}=h^{T} P_{V}^{\perp} d \omega_{d}+h^{T} P_{V}^{\perp} \delta
$$

The energy $E_{r}$ present in the estimated pixel vector of the filtered image can be expressed as the sum of vector components squared or the inner product of the pixel vector with itself, $E_{r}=\left\langle h^{T} P_{V}^{\perp} \hat{r}\right\rangle^{2}$. Similarly, the energy of the objected scattering mechanism and the lack-of-fit error are given by $E_{d}=\left\langle h^{T} P_{V}^{\perp} d \omega_{d}\right\rangle^{2}$ and $E_{e}=\left\langle h^{T} P_{V}^{\perp} \delta\right\rangle^{2}$, respective-ly. An energy ratio can be defined as

$$
\begin{aligned}
R & =\left\langle h^{T} P_{V}^{\perp} d \omega_{d}\right\rangle^{2} /\left\langle h^{T} P_{V}^{\perp} \delta\right\rangle^{2} \\
& =\frac{h^{T} P_{V}^{\perp} d \omega_{d}^{2} d^{T} P_{V}^{\perp} h}{h^{T} P_{V}^{\perp} E\left\{\delta \delta^{T}\right\} P_{V}^{\perp} h} .
\end{aligned}
$$

The expected value of $\delta \delta^{T}$ is $\sigma^{2} I$. Maximization of this quotient with respect to $h$ can be transformed into the generalized eigenvalue problem (J. W. V. Miller, et al. 1992). and the result of this maximization is $h^{T}=\alpha d^{T}$, where $\alpha$ is an arbitrary scalar. The weight of the objected scattering mechanism is obtained by substituting the value $h^{T}$ in (9) and we have

$$
\hat{\omega}_{d}(r)=\frac{d^{T} P_{V}^{\perp} \hat{r}}{d^{T} P_{V}^{\perp} d}
$$




\subsection{Interpretation of Utilized Subspace Projections}

The unique feature vectors of the objected and non-objected scattering mechanisms are linearly independent real-valued vectors in $\mathfrak{R}^{l \times 1}$, where $l$ is the dimension of feature space. A non-empty subset of $\mathfrak{R}^{l \times 1}$ is named a subspace. A set of all linear combinations of $\left(s_{1}, s_{2}, \ldots, s_{6}\right)$ is referred to the span of columns of $S$. This subspace is the subspace of interest in our target detection problem, because represents all of the possible mixture of scattering behaviors in the backscattering procedure. Consider that the entire feature space $\langle S\rangle$ is a set of all linear combinations of the feature vectors of the objected and nonobjected scattering mechanisms (columns of $S$ ). And also suppose that $\left\langle S_{d}\right\rangle$ and $\left\langle S_{n}\right\rangle$ are the spaces linearly spanned respectively by $S_{d}$ and $S_{n}$. As illustrated in Figure. 1, orthogonal projection $P_{S}$ projects estimated pixel vector onto entire feature space. Oblique projection $P_{S_{d} S_{n}}$ projects $\hat{r}$ along direction parallel to $\left\langle S_{n}\right\rangle$ onto $\left\langle S_{d}\right\rangle$. The non-objected scattering mechanisms will not be annihilated by orthogonal projection of the estimated pixel feature vector on $\left\langle S_{d}\right\rangle$. On the other hand, Orthogonal projection of $\hat{r}$ on $\left\langle S_{n}\right\rangle^{\perp}$ will corrupt $\left\langle S_{d}\right\rangle$. Therefore, projecting $\hat{r}$ obliquely onto $\left\langle S_{d}\right\rangle$ is the ideal case, and the vector resulting the multiplication of $P_{d S_{n}}$ and $\hat{r}$ will contain energy associated with $S_{d}$.

A pixel feature vector, which contains the objected scattering mechanism, is not completely represented by span of $S_{n}$ and thus has a non-zero oblique projection onto the target subspace. Operating the operator $P_{S_{d} S_{n}}$ on the mixed pixel represented by equation (6) we have

$$
P_{S_{d} S_{n}} \hat{r}=P_{S_{d} S_{n}} S_{d} \omega_{d}+P_{S_{d} S_{n}} S_{n} \omega_{n}+P_{S_{d} S_{n}} \delta
$$

The term $P_{S_{d} S_{n}} S_{n} \omega_{n}$ is clearly all zeros for all $\omega_{n}$ since $P_{S_{d} S_{n}}$ designates $\left\langle S_{n}\right\rangle$ as its null space, and the term $P_{S_{d} S_{n}} S_{d} \omega_{d}$ will be a non-zero vector which contains energy associated with the objected scattering mechanism.

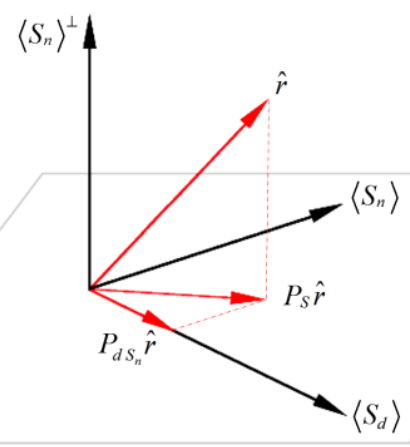

$\langle S\rangle=\left\langle S_{d} S_{n}\right\rangle$ space

Figure 1. The concept of the utilized subspace projections.

\section{VALIDATION}

To validate and test the potential of the detector, two full polarized datasets including a C-band Radarsat- 2 dataset acquired over San Francisco Bay and an L-band dataset acquired using uninhabited aerial vehicle SAR (UAVSAR) on the Southern California Coast, were chosen for the experiments. The Radarsat-2 dataset is single look complex (SLC) image and its nominal slant range resolution is about $5 \mathrm{~m}$ at near range to

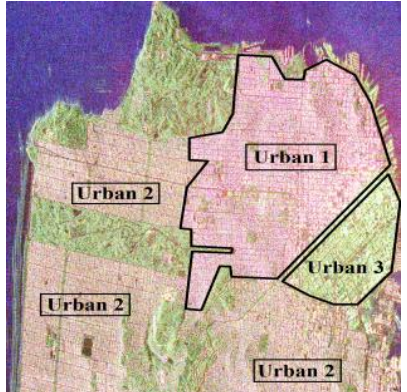

(a)

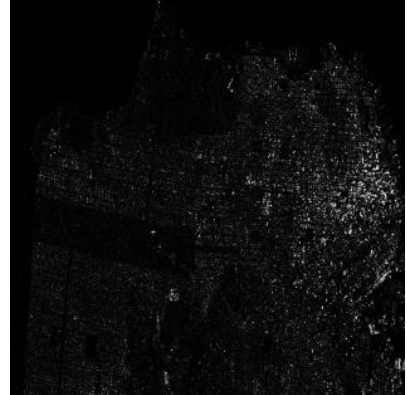

(b)

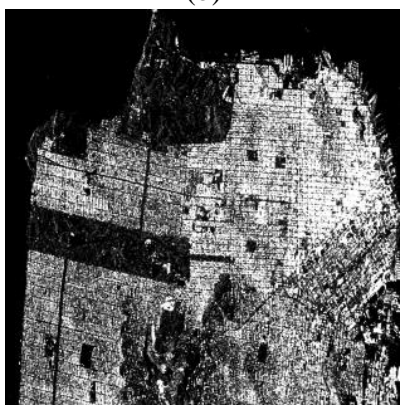

(c)

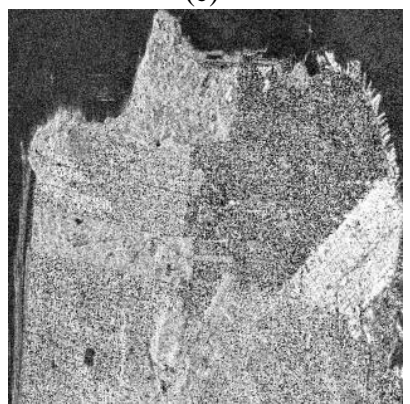

(d)

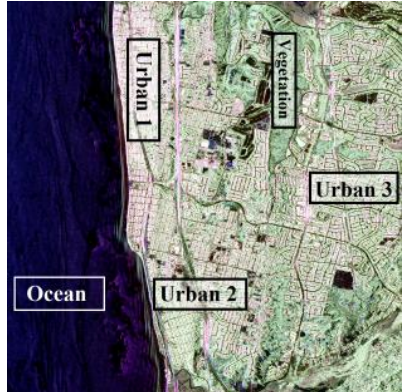

(e)

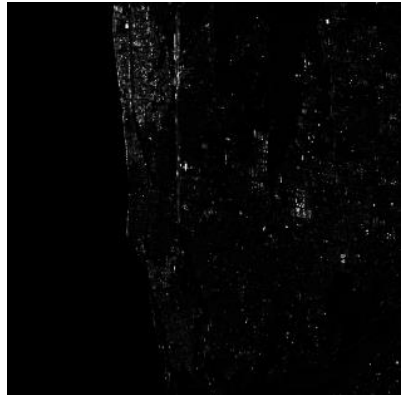

(f)

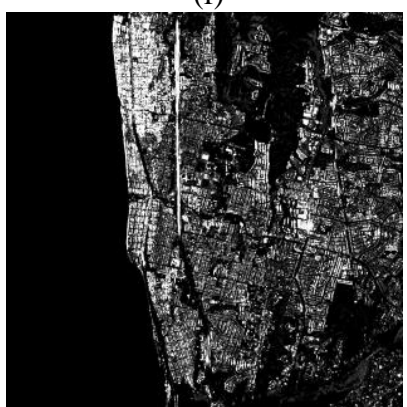

(g)

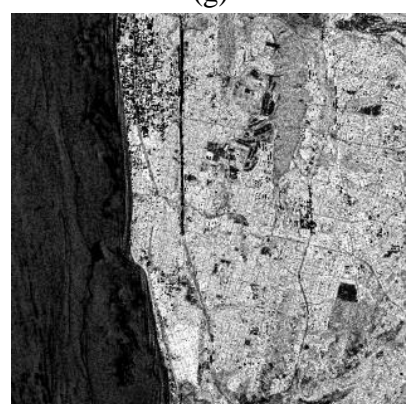

(h)
Figure. 1. San Francisco Bay study area: Pauli RGB image (a), left and right helix difference target map (b), detected built-up area (c), and helix scattering component of Yamaguchi target decomposition (d). Southern California Coast study area: Pauli RGB image (e), left and right helix difference target map (f), detected built-up area (g), and helix scattering component of Yamaguchi target decomposition (h).

$10.5 \mathrm{~m}$ at far range. The nominal resolution of the UAVSAR quad-polarimetric dataset is around $1.67 \mathrm{~m} \times 0.6 \mathrm{~m}$ (range $\times$ azimuth). The dataset is a multi-looked cross (MLC) product which was multilooked using an asymmetric window size of $3 \times 12$ (range $\times$ azimuth). It is expected that these two datasets, with different frequencies and resolutions, will return a broad picture of the detection capabilities of the proposed detector. In order to provide a closer inspection of the scenes the detections were performed on portions of the total images. For our analysis, the selected areas of interest are two sub-images of 
$1200 \times 1500$ and $1200 \times 1200$ pixels which were selected from the Radarsat-2 and UAVSAR datasets, respectively.

Figure. 2(a) and (e) presents the original span images and color composite RGB images of the aforementioned datasets where the three colors of colored images are the components of the Pauli vector. For urban areas in Figure. 2(a) and (e), due to different alignments of the buildings relative to the radar look direction, three built-up region themes were denoted by urban 1 , 2 and 3. As can be clearly observed, the buildings located in urban 1 region are perpendicular to the radar illumination direction, but man-made building structures located in urban 2 and 3 regions have a slightly and very oblique alignment to the radar azimuth direction, respectively.

A $3 \times 3$ moving averaging window is applied to obtain the local coherency matrix of the feature vector and the mean of the feature vector is calculated over the window. Figure. 2(c) and (g) present the sum of detected right and left helix detection results over San Francisco Bay and California Coast, respectively. Figure. 2(b) and (f), respectively, illustrate the difference of detected right and left helix detector magnitudes for San Francisco Bay and California Coast.

A look at Figure. 2(c) and (g) shows that almost all of the manmade building structures with any orientation were detected in urban 1, 2, and 3 regions. The buildings located in urban 1 regions due to their parallel alignment to radar azimuth direction (which makes significant fraction of energy to scattered backward) were detected with fairly large magnitude of detector and prominent in vision. Figure. 2(d) and (h) shows result of helix scattering component of Yamaguchi decomposition. From these figures, we can see that by detecting helix scattering mechanism under proposed detector the detection of built-up area is enhanced, and odd-bounce scattering mechanism is weakened. Especially, the contrast between built-up areas and other (vegetated, ocean, etc.) areas is greatly improved.

\section{CONCLUSION}

This paper presented a study that characterizes urban scattering from target detection point of view. This is a relatively new research field for urban remote sensing. From the above discussion, it can be concluded that the proposed feature scattering vector formalism is effective for analysis of target characteristics and target detection. We studied how to use right and left helix scattering mechanism under proposed feature vector formalism may improve urban characterization. We showed that helix scattering mechanism detection under the proposed detection procedure have a more significant influence in distinguishing between natural and man-made objects. This stresses the fact, an accurate characterization of stable feature in urban environment is possible even with low resolution spaceborne radar platforms.

\section{REFERENCES}

J. Gu, J. Yang, H. Zhang, Y. Peng, C. Wang, and H. Zhang, "Speckle filtering in polarimetric SAR data based on the subspace decomposition," IEEE transactions on geoscience and remote sensing, vol. 42, pp. 1635-1641, 2004.

J.-S. Lee and E. Pottier, Polarimetric radar imaging: from basics to applications: CRC press, 2009.

J.-S. Lee and K. Hoppel, "Principal components transformation of multifrequency polarimetric SAR imagery," IEEE Transactions on Geoscience and Remote Sensing, vol. 30, pp. 686-696, 1992.
J. W. V. Miller, J. B. Farison, and Y. Shin, "Spatially invariant image sequences," IEEE Trans. Image Processing, vol. 1, pp. 148- 161, Apr. 1992.

S. Cloude, Polarisation: applications in remote sensing: Oxford University Press, 2010

S.-W. Chen, Y.-Z. Li, X.-S. Wang, S.-P. Xiao, and M. Sato, "Modeling and interpretation of scattering mechanisms in polarimetric synthetic aperture radar: Advances and perspectives," IEEE Signal Processing Magazine, vol. 31, pp. 7989, 2014. 